www.nature.com/pj

\title{
Lower critical solution temperature behavior of poly(2-chloroethyl vinyl ether-alt-maleic anhydride) in organic media
}

Polymer Journal (2010) 42, 901-904; doi:10.1038/pj.2010.84; published online 8 September 2010

\section{INTRODUCTION}

The thermoresponsive behavior of the lower critical solution temperature (LCST) in an aqueous polymer solution is common and has been studied widely. However, it is rare for a polymer to exhibit a sensitive LCST in organic media. As a result of academic interest and industry applications, much attention has been drawn to thermoresponsive polymers in organic media. In the past, a few studies $^{1-8}$ investigated LCST-type phase separation in organic solvents under tough conditions, such as at a high pressure above the boiling point of the solvent in a sealed cell. While the temperature increases, the difference in the size or expansion coefficient between the polymer and solvent also increases and causes phase separation at a certain critical temperature, as explained by the theory of free volume. ${ }^{9}$ Although some theories and models ${ }^{9-14}$ have tried to elucidate and predict the phase behavior of polymer/organic solvent systems, until now novel LCST-type systems of polymers in organic media have seldom been identified, especially in mild conditions. ${ }^{15-17}$

In this paper, experimental observations of unique phase behavior in a poly(2-chloroethyl vinyl ether-alt-maleic anhydride) solution are reported. In some organic solvents, the polymer presents LCST-type thermoresponsive phase behavior under mild conditions. The clouding temperature of the polymer solution can be tuned easily in a wide range with different media. In some organic media, the polymer solution can form a relatively stable dispersion system above a certain temperature. This dispersion system can recover to become a dilute polymer solution as the temperature of the system falls below the clouding temperature.
This kind of process is reversible and very similar to the behavior of poly $(N$-isopropyl acrylamide) aqueous solutions.

\section{EXPERIMENTAL PROCEDURE}

Copolymerization of maleic anhydride and 2-chloroethyl vinyl ether was carried out by free-radical solution polymerization. Typically, maleic anhydride (1.225 g), 2-chloroethyl vinyl ether (1.332 g), 2,2'-azobisisobutyronitrile (AIBN) $(0.006 \mathrm{~g})$ and methyl ethyl ketone (5g) were added into a 50-ml three-neck flask equipped with a condenser and an inlet of nitrogen gas. The reaction solution was stirred for $2 \mathrm{~h}$ at $70{ }^{\circ} \mathrm{C}$. The resultant polymer was precipitated in diethyl ether and dried under vacuum at $100{ }^{\circ} \mathrm{C}$ for at least $8 \mathrm{~h}$.

It is well known that vinyl ethers and maleic anhydride can form alternating copolymers with a 1:1 overall composition by free-radical polymerization. ${ }^{18}$ The ${ }^{1} \mathrm{H}$ and ${ }^{13} \mathrm{C}$ NMR spectra (see Supporting Information, Figure S1) suggested that the copolymer of 2-chloroethyl vinyl ether and maleic anhydride is an alternating polymer because the signals from the main chain methylene carbon appeared around 32-37 p.p.m. in the ${ }^{13} \mathrm{C}$ NMR spectrum. ${ }^{18}$

The weight-averaged molecular weight and molecular weight distribution index $M_{\mathrm{w}} / M_{\mathrm{n}}$ for the obtained copolymer were $66000 \mathrm{~g} \mathrm{~mol}^{-1}$ and 3.14, respectively, as determined using a gel permeation chromatogram using tetrahydrofuran (THF) as the eluent and standard polystyrene for molecular weight calibration (see Supporting Information, Figure S2).

The thermoresponsive phase behavior of the polymer solution was characterized using a static light scattering (SLS) technique. The sample solution was optically purified with a membrane filter (Millipore, MA, USA) with a nominal pore size of $1.0 \mu \mathrm{m}$ and transferred to an optical tube. The sealed tube was kept in a refrigerator at about $3{ }^{\circ} \mathrm{C}$ overnight before SLS measurements. The scattered light intensity at different temperatures at a fixed angle of $90^{\circ}$ was measured with a laboratory-made apparatus equipped with an ALV/SO-SIPD detector (ALV, Langen, Germany) using a He-Ne laser (wavelength $\lambda_{0}=633 \mathrm{~nm}$ ). ${ }^{19}$ The heating and cooling rates of the system were about $0.2^{\circ} \mathrm{C} \mathrm{min}{ }^{-1}$. The scattered light intensity of the polymer solution was measured immediately when the system reached the desired temperature. The point at which the intensity of the scattered light increased sharply was considered the clouding temperature of the polymer solution.

\section{RESULTS AND DISCUSSION}

In the $\mathrm{THF} / \mathrm{h}$ exane mixed solvent with proper composition, the dilute solution of poly(2-chloroethyl vinyl ether-alt-maleic anhydride) exhibited an abrupt increase in the scattered light intensity at a certain temperature (Figure 1). At a higher temperature, the polymer solution became turbid, as shown in Figure 2. This turbid solution became transparent again as the system cooled down. That is to say, the polymer became insoluble above a critical temperature, and phase separation was observable by eye in the final stage. In other words, poly (2-chloroethyl vinyl ether-alt-maleic anhydride) presents LCST-type thermoresponsive phase behavior in organic media such as THF/ hexane under relatively mild conditions, that is, much lower than the boiling point of the solvent. With the increase in THF in the mixed solvent, the clouding temperature of the polymer solution increased. As the 


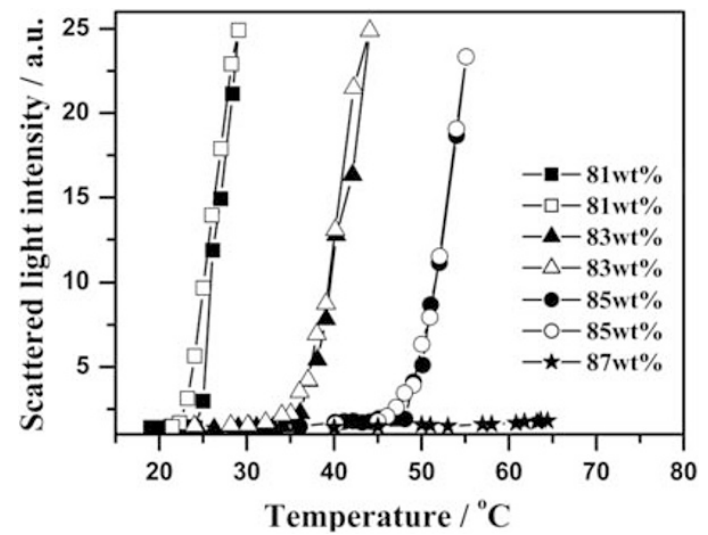

Figure 1 Temperature dependence of the scattered light intensity for poly(2-chloroethyl vinyl ether-altmaleic anhydride) solutions in mixed solvents of THF and hexane. The weight percentage of THF is indicated in the graph, and the polymer concentration in the solution is about $0.1 \mathrm{wt} \%$. The solid and hollow symbols represent heating and cooling processes, respectively.

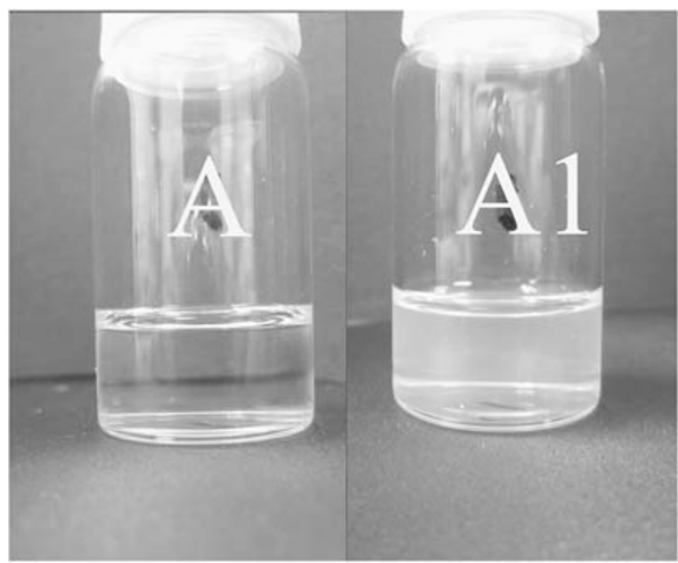

Figure 2 Photograph of the polymer solution in THF/hexane with $81 \mathrm{wt} \%$ THF at different temperatures: $\mathrm{A}, 20^{\circ} \mathrm{C} ; \mathrm{A} 1,40^{\circ} \mathrm{C}$.

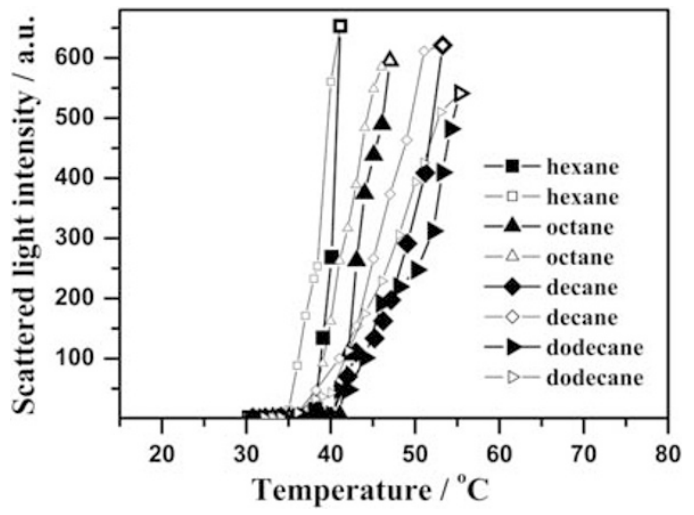

Figure 3 Temperature dependence of scattered light intensity for the polymer solution in mixed solvents of propyl acetate and various $n$-alkanes with $83 \mathrm{wt} \%$ propyl acetate. The solid and hollow symbols represent heating and cooling processes, respectively.

concentration of the polymer in the solvent was low (about $0.1 \mathrm{wt} \%$ ), the cloud point temperature was observed by eye to be higher than the critical solution temperature as measured by SLS. For example, in the mixed solvent with $81 \mathrm{wt} \%$ THF shown in Figure 2, the visually observed clouding temperature is about $40^{\circ} \mathrm{C}$, which is higher than the critical temperature of $25^{\circ} \mathrm{C}$ measured by SLS. It can also be seen from Figure 1 that there exists a discrepancy in the temperature dependence of the scattered light intensity when the cooling curve is compared with the heating curve at a lower weight percentage of THF in the mixed solvent.

The thermoresponsive behavior of the polymer in the mixed solvent of propyl acetate and various $n$-alkanes was also observed and was reversible (Figure 3 ). It can be seen from Figure 3 that the slope of the curves becomes flatter with increasing $n$-alkane size; that is, the sensitivity of the solution turbidity against temperature becomes weaker with increasing $n$-alkane size. It is obvious that the increase rate of scattered light intensity of the polymer solution in the mixed solvent of propyl acetate, $n$-dodecane and $n$-decane (about 52 a.u. per ${ }^{\circ} \mathrm{C}$ ) is slower than that in other mixed solvents containing shorter $n$-alkanes (at least 60 a.u. per ${ }^{\circ} \mathrm{C}$ ). This tendency also exists during the cooling process. There is also a discrepancy between the curves for heating and cooling in these mixed solvents.

The clouding temperature of the polymer solution decreased as the weight of 1,2dichloroethane increased in its mixture with propyl acetate (Figure 4). The discrepancy between the heating and cooling processes becomes remarkable as the weight percentage of propyl acetate in the mixed solvent decreases.

For poly(2-chloroethyl vinyl ether-alt-maleic anhydride), THF and propyl acetate are good solvents, and $n$-alkane and 1,2-dichloroethane are nonsolvents. As the nature of the media becomes poor, that is, with increased levels of nonsolvents, the clouding temperature of the polymer solution decreases, and the discrepancy between heating and cooling scans becomes more pronounced. A more remarkable discrepancy during cooling is observed in the mixed solvent of butyl acetate/ $n$-octane, and a much longer time is needed to recover to the original transparent state (Figure $5, a \rightarrow b$ ). It can be concluded that butyl acetate is not a very good solvent, especially at higher temperatures, although the polymer can be dissolved in it. Thus, SLS was measured only for butyl acetate. LCST-type phase behavior was observed, as shown in curve $\mathrm{C}$ in Figure 5. The clouding temperature of the polymer in butyl acetate is high (about $95^{\circ} \mathrm{C}$ ) but far enough below its boiling temperature $\left(124-126^{\circ} \mathrm{C}\right)$ and discrepancies are less pronounced.

One of the reasons that alternating copolymers of 2-chloroethyl vinyl ether and maleic anhydride exhibit LCST-type behavior may 


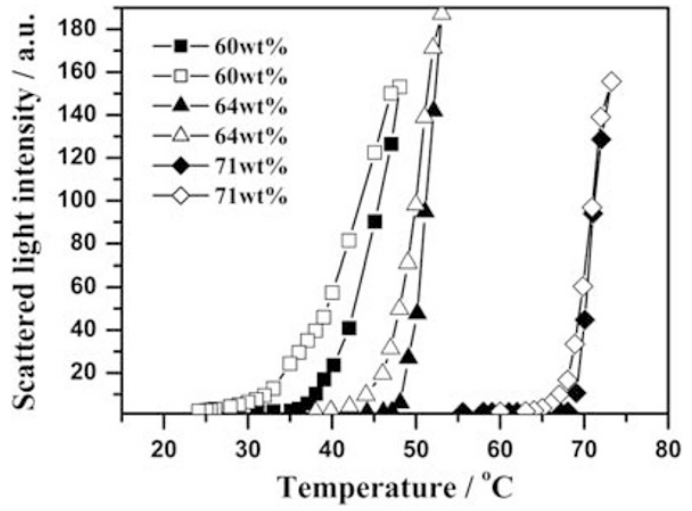

Figure 4 Temperature dependence of scattered light intensity for polymer solutions in mixed solvents of propyl acetate and 1,2-dichloroethane. The weight percentage of propyl acetate in the mixed solvents is indicated in the graph. The solid and hollow symbols represent heating and cooling processes, respectively.

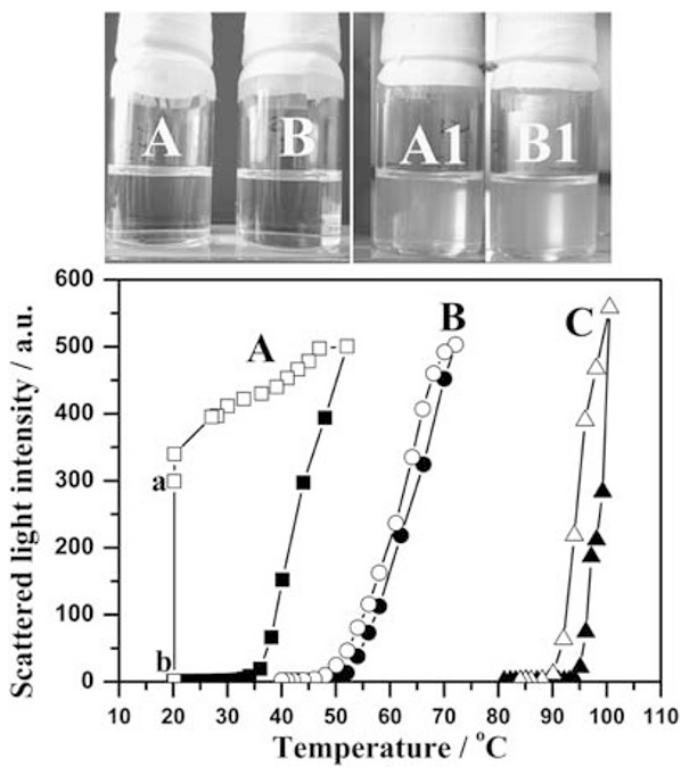

Figure 5 Temperature dependence of the scattered light intensity for polymer solutions in different organic media: (A) heating (solid square) and cooling (hollow square) processes in the mixed solvent of butyl acetate and $n$-octane with $83 \mathrm{wt} \%$ butyl acetate. Data points a and b represent the results for 1 and $3 \mathrm{~h}$ after finishing the cooling process, respectively; (B) heating (solid circle) and cooling (hollow circle) processes in the mixed solvent of ethyl acetate and $n$-octane with $83 \mathrm{wt} \%$ ethyl acetate; (C) heating (solid triangle) and cooling (hollow triangle) processes in butyl acetate. In the upper photograph, bottles $\mathrm{A}$ and $\mathrm{B}$ represent the polymer solutions of butyl acetate/n-octane and ethyl acetate/n-octane, respectively, at $20^{\circ} \mathrm{C}$. These solutions become turbid after heating to $70^{\circ} \mathrm{C}$, as shown in $\mathrm{A} 1$ and $\mathrm{B} 1$.

be the existence of unique interactions between the polymer and solvent because of the unique chemical composition of these polymers. In our preliminary experiments, some polymers with a similar chemical structure did not exhibit LCST behavior in some related organic media; for instance, poly(ethyl vinyl ether-alt-maleic anhydride) did not exhibit any LCST behavior under the same conditions. Unfortunately, it is difficult to prepare random copolymers of 2-chloroethyl vinyl ether and maleic anhydride; hence, the influence of the monomer sequence cannot be evaluated. In this kind of thermoreversible system, conformation of polymer in the solvent and the morphology and stability of the dispersed particles change during phase separation, which could help us to understand the polymer solution's chemistry more accurately. Furthermore, this kind of polymer, with groups of carboxylic anhydride and alkyl chloride, can be modified conveniently. Therefore, on the basis of the novel LCST thermosensitivity of this polymer, self-assembly of the polymer with different compositions or topology could be studied, and some novel sensors or smart materials could be developed.

In conclusion, LCST-type phase behavior in poly(2-chloroethyl vinyl ether-alt-maleic anhydride) can be obtained in the proper organic media under mild conditions, and the clouding temperature of the polymer solution can be tuned conveniently in a wide range. It should be emphasized that this copolymer does not contain any polar groups, and the solvents used are nonpolar. Further investigations of LCST behavior at different polymer concentrations using samples of various molecular weight are in progress.

Zhenjie Liu ${ }^{1}, \begin{array}{r}\text { Yongliang Guo } \\ \text { Katsuhiro Inomata }\end{array}$
${ }^{1,2}$ and
Department of Materials Science and
Engineering, Nagoya Institute of
Technology, Gokiso-cho, Nagoya, Japan
and ${ }^{2}$ Department of Materials Science
and Engineering, Beijing University of
Chemical Technology, Beijing, China
E-mail: inomata.katsuhiro@nitech.ac.jp

1 Patterson, D., Delmas, G. \& Somcynsky, T. A comparison of lower critical solution temperature of some polymer solutions. Polymer 8, 503-516 (1967).

2 Burchard, W. Thermodynamic solution properties of three polymers exhibiting lower critical solution temperature. Polymer 10, 467-475 (1969).

3 Cowie, J. M. G., Maconnachie, A. \& Ranson, R. J. Phase equilibria in cellulose acetate-acetone solutions. The effect of the degree of substitution and molecular weight on upper and lower critical solution temperatures. Macromolecules 4, 57-61 (1971).

4 Siow, K. S., Delmas, G. \& Patterson, D. Cloud-point curves in polymer solutions with adjacent upper and lower critical solution temperatures. Macromolecules $\mathbf{5}$, 29-34 (1972).

5 Saeki, S., Kuwahara, N., Konno, S. \& Kaneko, M. Upper and lower critical solution temperatures in polystyrene solutions. Macromolecules 6, 246-250 (1973).

6 Konno, S., Saeki, S., Kuwahara, N., Nakata, M. \& Kaneko, M. Upper and lower critical solution temperatures in polystyrene solutions. iv. role of configurational heat capacity. Macromolecules 8, 799-804 (1975).

7 Cowie, J. M. G. \& Swinyard, B. Location of three critical phase boundaries in poly(acrylic acid)-dioxane solutions. Polymer 31, 1507-1513 (1990).

8 Bae, Y. C., Lambert, S. M., Soane, D. S. \& Prausnitz, J. M. Cloud-point curves of polymer solutions from thermooptical measurements. Macromolecules 24, 4403-4407 (1991).

9 Patterson, D. Free volume and polymer solubility. Macromolecules 2, 672-677 (1969).

$10 \mathrm{Ho}$, C. B. \& Chan, B. Y. Liquid-liquid equilibria of binary polymer solutions with specific interactions. Polymer 39, 6449-6454 (1998).

11 Wang, F., Saeki, S. \& Yamaguchi, T. Absolute prediction of upper and lower critical solution temperatures in polymer/solvent systems based on corresponding state theory. Polymer 40, 2779-2785 (1999).

12 Jang, J. G. \& Bae, Y. C. Phase behavior of binary polymer solutions: the extended lattice fluid model. J. Appl. Polym. Sci. 70, 1143-1150 (1998). 
13 Liu, H. \& Zhong, C. Modeling of the $\theta$ (lower critical solution temperature) in polymer solutions using molecular connectivity indices. Eur. Polym. J. 41, 139-147 (2005).

14 Nies, E., Stroeks, A., Simha, R. \& Jain, R. K. LCST phase behavior according to the simha-somcynsky theory: application to the $n$-hexane polyethylene system. Colloid Polym. Sci. 268, 731-743 (1990).

15 Seno, K.- I., Kanaoka, S. \& Aoshima, S. Synthesis and LCST-type phase separation behavior in organic solvents of poly(vinyl ethers) with pendant imidazolium or pyridinium salts. J. Polym. Sci. A Polym. Chem. 46, 5724-5733 (2008).

16 Mugisawa, M., Ohnishi, K. \& Sawada, H. Preparation of novel fluoroalkyl-end-capped 2-acrylamido-2-methylpropanesulfonic acid cooligomeric nanoparticles containing adamantane units possessing a lower critical solution temperature characteristic in organic media. Langmuir 23, 5848-5851 (2007).

17 Kuroiwa, K., Shibata, T., Takada, A., Nemoto, N. \& Kimizuka, N. Heat-set gel-like networks of lipophilic co(ii) triazole complexes in organic media and their thermochromic structural transitions. J. Am. Chem. Soc. 126, 2016-2021 (2004).

$18 \mathrm{Ha}$, N. T. H. Fujimori, K. Henry, P. C. \& Tucker, D. J. Assignment of ${ }^{13} \mathrm{C}$ NMR chemical shift and microstructure of copolymers of 2-chloroethyl vinyl ether-maleic anhydride and n-butyl vinyl ether-maleic anhydride. Polym. Bull. 43, 81-85 (1999).

19 Inomata, K., Kasuya, M., Sugimoto, H. \& Nakanishi, E. Effect of helix-coil transition on association behavior of both-ends hydrophobically-modified water-soluble polypeptide. Polymer 46, 10035-10044 (2005).

Supplementary Information accompanies the paper on Polymer Journal website (http://www.nature.com/pj) 